\title{
Oleuropein: Methods for extraction, purifying and applying
}

\author{
Deborah Murowaniecki Otero ${ }^{1 * \mathbb{D}}$, Fernanda Moreira Oliveira ${ }^{2}$, Alexandre Lorini ${ }^{2}$, Bruna da Fonseca Antunes ${ }^{3}$, \\ Raquel Moreira Oliveira ${ }^{3}$, Rui Carlos Zambiazi ${ }^{3}$
}

10.1590/0034-737X202067040009

\begin{abstract}
Oleuropein is one of the most abundant phenolic compounds present in olive leaves, and many studies have shown that this compound has important biological properties (anti-inflammatory, anti-atherogenic, anticancer, antimicrobial and antiviral) and that is why it has been gaining prominence in research. Oleuropein can be extracted from different sources using standard and unconventional methods, and can be recovered and purified (mainly by chromatographic techniques), for later use in several areas. The literature presents information about oleuropein alone in scientific research with different objectives, however, it was observed by the authors that studies that compile the existing information on these secoiridoides are very scarce and, therefore, this review was developed with the aim of providing current information to the scientific community, about the different techniques of extraction and purification of oleuropein, as well as the opportunities for applications and uses of this compound.
\end{abstract}

Keywords: phenolic compound; extraction; olive leaves; review.

\section{INTRODUCTION}

Oleuropein, characterized as a bitter glycoside, is one of the most abundant phenolic compounds in olive leaves, and it is also present in the trunk and the fruit of the olive tree (skin, pulp and stone) (Romero et al., 2017). Several studies have indicated that this phenolic compound presented interesting biological and pharmacological properties, in large part attributed to its putative antioxidant and anti-inflammatory effects (Cavaca et al., 2020), antimicrobial properties (Guinda, 2006; Tripoli et al., 2005; Bayram et al., 2020), anticancer potential (Bonoli et al., 2004; Przychodzen et al., 2019); cardioprotection activity (Papachristodoulou et al., 2019; Nediani et al., 2019); affects lipid metabolism (Fki et al., 2020; Ucella, 2001), reduce body weight, alleviated kidney injury, and decreased oxidative stress and inflammatory response and inhibits platelet aggregation (Liu et al., 2019) and reduces LDL levels (Hadrich et al., 2016).

Its ability to act as a natural antioxidant has also attracted the attention of researchers, as consumers increasingly seek natural products or products that contain natural components in their formulation, inducing the industry to replace synthetic antioxidants with those extracted from plant sources (Mosca et al., 2013).

The choice of method of extraction and purification is considered one of the most critical stages of research involving natural products. The efficiency of the extraction process depends on several parameters, such as the type of sample, the compound to be extracted, its location in the plant material (Mustafa \& Turner, 2011), besides the type of solvent (Xynos et al., 2012), time and temperature of the extraction (Galanakis et al., 2010), and the methods

\footnotetext{
Submitted on July $11^{\text {th }}, 2019$ and accepted on July 09th 2020

${ }^{1}$ Universidade Federal da Bahia, Escola de Nutrição, Departamento de Ciência dos Alimentos, Salvador, Bahia, Brazil. deborah.m.otero@gmail.com

${ }^{2}$ Universidade Federal de Pelotas, Faculdade de Agronomia Eliseu Maciel, Departamento de Ciência e Tecnologia Agroindustrial, Pelotas, Rio Grande do Sul, Brazil. fer.moroli @ gmail.com; alexandrelorini@hotmail.com

${ }^{3}$ Universidade Federal de Pelotas, Centro de Ciências Químicas, Farmacêuticas e de Alimentos, Pelotas, Rio Grande do Sul, Brazil, brunafonsecaantunes@gmail.com; raquelmoroli@gmail.com; zambiazi@gmail.com

*Corresponding author: deborah.m.otero@gmail.com
} 
of recovery and purification. Due to the importance of oleuropein in relation to several applications as well as the increase of research on the characteristics and applications of this compound, this review was elaborated with the objective of compiling the information available in the literature up to the present moment.

\section{Data Sources and Search Strategy}

A comprehensive review was conducted by systematically searching in PubMed and Springer Link for all studies that developed an a priori OBS until May 2019. Subject headings related to OBSs (Oleuropein, phenolic compound, olive leaves) and other key terms (extraction, application, purification) were considered. Exclusion criteria were articles that did not meet the inclusion criteria mentioned above; For a writing of this review a total of 113 references were selected which presented the information and data of interest to the authors.

\section{Oleuropein}

Oleuropein is the major phenolic constituent of the oil leaf (Olea europaea L.) and it is also present in olive oil and fruit, up to $14 \%$ of the dry weight (Bonechi et al., 2019). This compound consists of a molecule of elenolic acid linked to the orthodiphenol hydroxytyrosol by an ester bond and to a molecule of glucose by a glycosidic bond (Figure 1). The rupture of the glycosidic bond gives rise to glucose and the oleuropein aglycon that, under an aqueous environment, is rapidly transformed into the dialdehydic form of decarboxymethyl elenolic acid linked to hydroxytyrosol (Sivakumar et al., 2018) which is also a bitter substance.

The biosynthesis of oleuropein in Oleaceae (Figure 2) follow the mevalonic acid pathway, branching leads to biosynthesis of geraniol, 10 hydroxygeranoil, 10 hydroxynerol, and iridoidal. From iridoidal, loganin is biosynthesized, and later deoxyloganic acid, 7 epiloganic acid, and loganic acid are incorporated into ligustroside. Ligustroside is considered as a direct precursor of oleuropein, via 7 ketologanic acid as intermediate. A

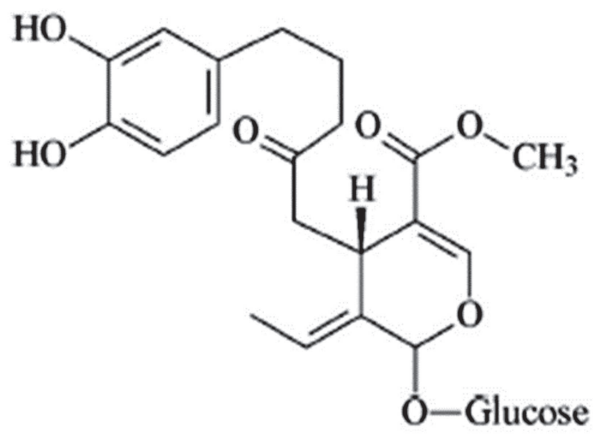

Figure 1: Structure oleuropein (Bonechi et al., 2019). probable biosynthetic route from deoxyloganic acid, 7 epiloganic acid, 7 ketologanic acid, 8 epikingisidic acid, oleoside 11 methyl ester, $7 \beta 1$ D glucopyranosyl 11 methyl oleoside, and ligustroside to oleuropein (Damtoft et al., 1992; Ahamad et al., 2019).

The enzyme involved in this reaction is â-glucosidase that rapidly acts during the crushing and malaxation steps of the olive oil extraction process to modulate the phenolic profile of virgin olive oil, and also contributes to the degradation of oleuropein at the beginning of the brining stage of natural table olives (Ramírez et al., 2016). Several factors influence the hydrolysis of oleuropein, such as $\mathrm{pH}$, type of organic acid, time as well as the interaction between these factors (Romero et al., 2020).

Oleuropein occurs in the Oleaceae, Gentianaceae and Cornaleae family and in lower concentrations in other plants and residues (olive pomace by e.g) (Böhmer-Maas et al., 2020) however the highest concentrations are described in olive leaves (60-90 mg. $\mathrm{g}^{-1}$ dry base) and young olives (63-105 mg.g $\mathrm{g}^{-1}$ dry base) (Tayoub et al., 2012). Oleuropein has several pharmacological properties, including antioxidant, anti-inflammatory, anti-atherogenic, anti-cancer, antimicrobial and antiviral, and for these reasons, it is commercially available as food supplement in Mediterranean countries (Omar, 2010). In view of all the reported benefits for oleuropein, it is necessary to conduct studies that seek new sources of the compound, as well as methods that provide better extractions and purifications of oleuropein, aiming at different application markets.

\section{Oleuropein extraction methods}

The extraction methods used for isolation of biomolecule from olive leaves show risk of residual solvent and less extraction efficiency. Hence, there is a need to develop novel techniques to encapsulate the risks headed with extraction process. Blanching of olive leaves causes deactivation of enzymes, and further exposure to ultrasonic waves enhances mass transfer of solvent and promotes the release of oleuropein. Hot blanching technique shows a significant linear upswing in the concentration of oleuropein when compared to direct extraction techniques (Sucharitha et al., 2019).

The extraction of oleuropein can be done in different ways, being classified in conventional and nonconventional (Figure 3), and these are described below.

In blanching, the thermal treatment applied to the olive leaves leads to structural changes of the plant tissue, being responsible for the loosens of the cellulosic networks; thereby, it promotes the leaching of contents from olive leaves. Extraction and isolation of the active moiety from the plant can be easily affected by the processing methods. Hence, suitable conditions are required to maximize the extraction efficiency and to

Rev. Ceres, Viçosa, v. 67, n.4, p. 315-329, jul/aug, 2020 
minimize the processing loss of phenolics. Sucharitha et al. (2019) studied the effect of three different techniques on the efficiency of oleuropein extraction. Hot blanching technique showed a significant linear upswing in the concentration of oleuropein when compared to direct extraction techniques.

\section{Conventional \\ Extraction by Soxhlet}

The Soxhlet extraction method is the one most commonly used in laboratories, but it may promote the partial degradation of thermolabile compounds, as well as consuming large amounts of water and energy (Bimakr et al., 2012).

When examining different extraction systems and factors such as solvent type, $\mathrm{pH}$ and temperature, it was found that the combination of solvents with water showed higher efficiency of extraction (the highest content of oleuropein (13 mg. $\mathrm{g}^{-1}$ dry leaf olive) was obtained using $80 \%$ ethanol, followed by $20 \%$ acetonitrile $\left(10 \mathrm{mg} \mathrm{g}^{-1} \mathrm{dry}\right.$ olive leaf powder). With this, the importance of water as a co-solvent to increase the amount of extracted oleuropein was confirmed (Yateem et al., 2014).

The elevation of the extraction temperature, showed a significant increase in the oleuropein content; and the increase in $\mathrm{pH}$ provided a reduction in extraction yield. In addition, the results showed that Soxhlet was more efficient in extracting oleuropein compared to the cold extraction technique (Yateem et al., 2014).

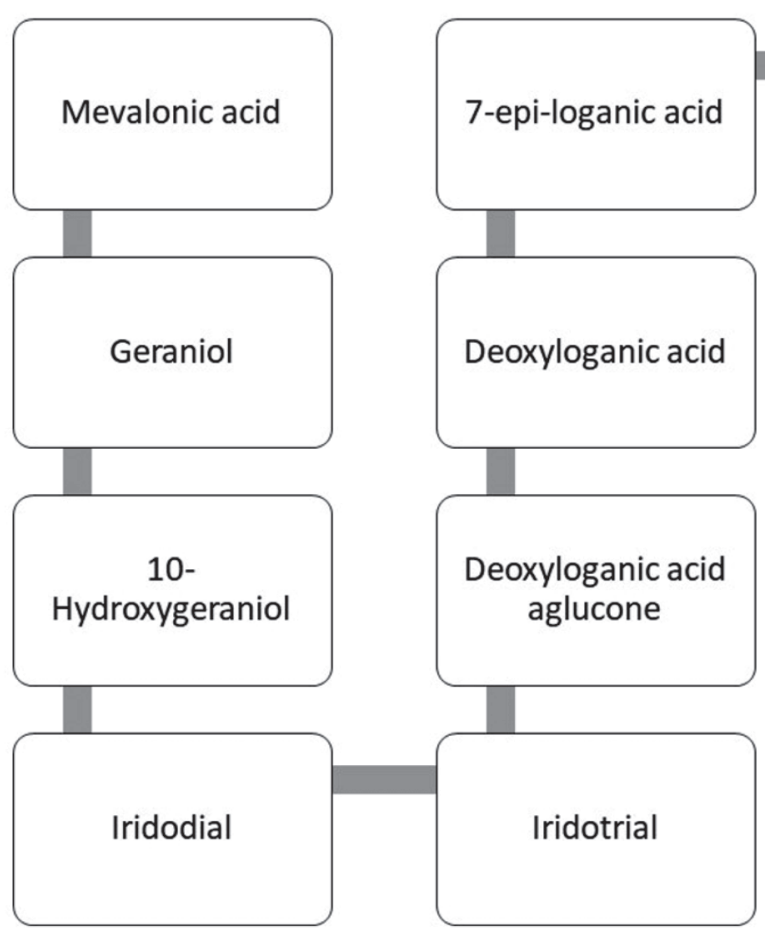

Figure 2: Biosynthesis of oleuropein in Oleaceae.
Yasemi et al. (2017) also evaluated different extraction methods, including maceration, soxhlet, ultrasound and microchannel, with soxleth extraction showing the greatest recovery among conventional techniques (62\%). In addition, different combinations of solvents were also tested, where for soxleth the best combination was ethanol: water (80:20). However, the emerging techniques showed greater extraction yields (values between 80 and 95\%) compared to the conventional extraction methods evaluated by these researchers.

\section{Cold Solvent Extraction}

In this technique, also known as maceration, the sample is immersed in a non-volatile solvent at room temperature. Extraction can be done using water (Ghomari et al., 2019; Benincasa et al., 2019; Ghelichkhani et al., 2019) or alcoholic solvents (Bayraktar et al., 2019; Kabbash et al., 2019; Ghomari et al., 2019). Ethanol, methanol, ethyl acetate, water, hexane, diethyl ether and acetone have been used as solvents. Methanol seems to be a very effective solvent, yet its use may result in undesirable toxic residues in the final extracts (Lamprou et al., 2020).

It is a time-consuming process, which may lead to incomplete extraction, besides facing the probability of contamination when using solvents containing large amounts of water (Melecchi et al., 2002; Miranda \& Cuéllar, 2001).

Coppa et al. (2017) were able to obtain a lyophilized extract from olive leaves, which contained approximately

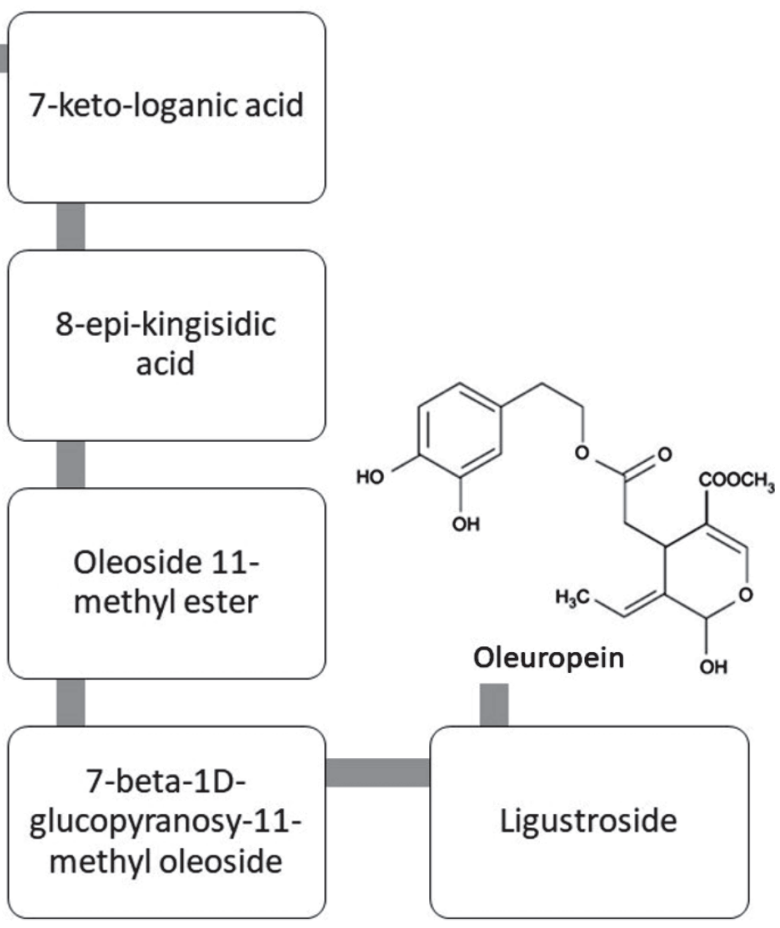


$18 \mathrm{~g} .100 \mathrm{~g}^{-1}$. These authors used maceration at $25^{\circ} \mathrm{C}$ for 2 $\mathrm{h}$ with a mixture of ethanol and water (70:30 v/v) and 1\% acetic acid, and observed that the highest oleuropein content was reached with an olive leaf: solvent ratio equal to $1: 3$, while the proportions 1: 8 and 1: 6 were also tested, with or without acetic acid present.

Cho et al., (2020) determined optimal conditions for olive leaf extraction by examining the effect of extraction solvent (water, aqueous methanol, aqueous ethanol, and aqueous acetone) on selected extract properties. The highest extraction efficiency of $20.41 \%$ was obtained using 90 vol\% methanol.

\section{Non-conventional methods}

The production of bioactive compounds from olive leaves requires treatments capable of breaking the lignocellulosic structure. Current research focuses on use of inexpensive, quick, and not harmful to the environment treatments, searching a more simplified large-scale operation approach (Lama-Muñoz et al., 2019). Nonconventional treatments such as ultrasound, supercritical fluid, pressurized fluid, microwave assisted, microchannels and membranes are highlighted in this scenario.

\section{Ultrasound assisted extraction}

Recently, advances in applied chemistry have led to possible new emerging industrial techniques like ultrasound-assisted extraction (UAE). This technology is a promising candidate as a green treatment solution for olive leaves utilization in a biorefinery. However, this application goes through prior optimization of technique and operating conditions (Lama-Muñoz et al., 2019).

Ultrasound-assisted extraction has the advantage of reducing the extraction time of the component of interest, mainly due to the physical and chemical effects promoted by cavitation, a phenomenon that accelerates the chemical reactions (Soria \& Villamiel, 2010). This method has been described by several authors (Shirzad et al., 2017; Cifá et al., 2018; Irakli et al., 2018; Giacometti et al., 2018) with the aim of extracting oleuropein from olive leaves.

In this phenomenon, the formation and collapse of cavitation bubbles occur, creating discrete areas of high pressure and temperature, which contribute to the extraction of the compounds (Bendicho et al., 2012). In addition, the ultrasound system allows for a reduction in the volume of chemical solvents, as it is a simple and effective process (Charpe \& Rathod, 2012). A disadvantage of this process is the possible damage that can be caused by free radicals (Fellows, 2009). When used in plants, the ultrasonic process favors the dilation and hydration of the plant material and causes the enlargement of the pores of the cell wall. This leads to an increase in swelling and mass transfer ratio and, eventually, to cell wall breakdown, and in this case causes increased solvent penetration (Toma et al., 2001; Chung et al., 2010).

Zun-Qiu et al. (2015) obtained $13.52 \%$ pure oleuropein from olive leaves by means of ultrasound-assisted extraction using a solution of $80 \%$ methanol-water (v/v). The same authors obtained oleuropein with a purity of $96.54 \%$ and with purification efficiency of $78.49 \%$, using

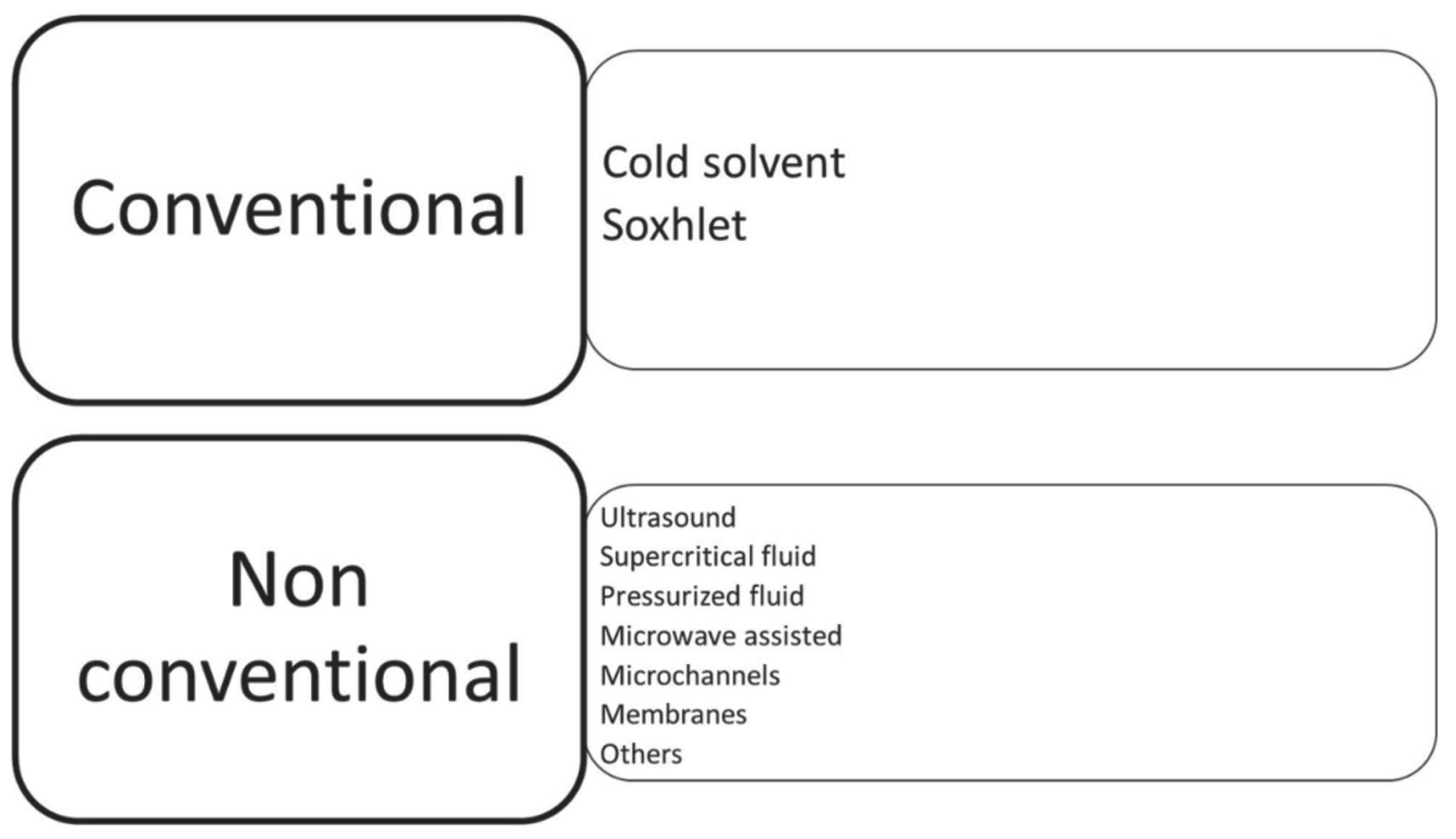

Figure 3: Main methods of extracting oleuropein.

Rev. Ceres, Viçosa, v. 67, n.4, p. 315-329, jul/aug, 2020 
silica gel column chromatography. Their study aimed to investigate the changes in oleuropein and total phenolic content over a year, as well as evaluating the antioxidant activity of oleuropein in vitro. The authors obtained different levels of oleuropein in 29 varietals cultivated in a region of China that is important for olive cultivation, but both had similar trends throughout the year. Samples harvested in January showed a higher content of oleuropein, and the lowest value was observed in samples collected in July. During the period of differentiation of flower buds and fruit development, a reduction in oleuropein content was observed.

Khemakhem et al. (2017) observed that the extraction kinetics of oleuropein from olive leaves improved with increasing temperature, using ultrasound-assisted extraction and water as solvent. This process also enhanced the initial extraction rate, with approximately $88 \%$ oleuropein extracted in the first minute of the experiment lasting 10 min, reaching $6.57 \pm 0.18 \mathrm{~g} .100 \mathrm{~g}^{-1}$ at $50^{\circ} \mathrm{C}$.

Lama-Muñoz et al. (2019) studied the optimization of extraction of oleuropein from olive leaves through an investigation of the influence of key factors of ultrasoundassisted extraction using an experimental central composite design, in comparison with conventional Soxhlet extraction. The highest extraction efficiency and antioxidant capacity were obtained under optimal increment of temperature and amplitude conditions $\left(40{ }^{\circ} \mathrm{C}\right.$ and $30 \%$, respectively).

Del Mar-Contreras et al. (2020) obtained an extract with both high level of oleuropein was achieved using the following operational parameters: solid-to-liquid ratio, 5.9\%; ethanol concentration, $47 \%$; extraction time, $50 \mathrm{~min}$.

\section{Extraction with supercritical fluid}

Substances in the supercritical state have intermediate properties between the properties of a gas and a liquid. With diffusion coefficient, low viscosity and absence of surface tension, referring to the characteristics of a gas, supercritical fluids have a greater diffusion capacity than liquid ones, thus allowing a fast and efficient transfer of mass and ease of penetration into a solid matrix. The density of these fluids is close to that of a liquid, providing good solvent power (Taylor, 1996; Luque De Castro, 2004). The technique of supercritical fluid extraction is commonly used for thermally sensitive analytes, since one of its advantages over conventional methods is the use of low temperatures. Other advantages are low energy consumption and low levels of degradation of chemical compounds; moreover, products of higher quality are obtained, in addition to high selectivity by polar analytes. In this technique, operational changes during extraction are allowed, facilitating the recovery of specific compounds (Reverchon \& De Marco, 2006).
The most commonly used solvent for extraction in supercritical media is carbon dioxide $\left(\mathrm{CO}_{2}\right)$, which is inert, non-flammable and does not harm the environment (Jarvis \& Morgan, 1997) however, this substance has an apolar nature, making it impossible to use in extracting polar compounds (Duarte, 2011). Also, because high pressure is used, expensive equipment is needed, increasing the cost of the final product (Herrero et al., 2006).

The extraction of oleuropein from olive leaves using method supercritical fluid has been described by some authors (Xynos et al., 2012; Bastante et al., 2018; Baldino et al., 2018). In the development of a green extraction procedure with supercritical fluid to produce extracts enriched with oleuropein from olive leaves, Xynos et al. (2012) acquired $30 \%$ of this compound in the dry extract, using $20 \%$ of ethanol as co-solvent and supercritical $\mathrm{CO}_{2}$ at 300 bar. This $30 \%$ was equivalent to the recovery of $5.1 \%$ of the original content of oleuropein in olive leaves.

\section{Extraction with pressurized fluid}

Extraction with pressurized fluid, also known as pressurized solvent extraction or accelerated solvent extraction, allows extraction in a closed and inert environment, under pressures not exceeding 200 bar and temperatures between 25 and $200^{\circ} \mathrm{C}$. In this process, the pressurized solvents remain in the liquid state, even in temperatures above boiling point, allowing extraction at high temperatures. Thus, inert solvents, such as ethanol and water, which are used in the extraction of some phenolic compounds at low temperatures, can be very efficient at high temperatures applied in the extraction with pressurized fluid. This technique allows rapid extraction and reduction of solvent consumption, and has therefore been successfully used in the extraction of several classes of compounds in plants (Santos et al., 2012; Jaski et al., 2019).

Xynos et al. (2014) applied pressurized liquid extraction to optimize extraction yield of the oleuropein content of leaves of $O$. europea, and to analyze the antioxidant activity of the extracts. The maximum content of oleuropein in leaf extract was $26.1 \%$, and this content was influenced mainly by three factors, in order of statistical significance: ethanol concentration - with positive effect; temperature - with negative effect; and extraction cycles - with positive effect. The highest yield was $46.64 \%$, and the antioxidant activity was not statistically significant when correlated with the phenolic content of leaf extracts.

The pressurized liquid extraction (PLE) is an alternative extraction technique to supercritical fluid extraction (SFE), investigated by Rosa et al., (2019) and proved adequate for the extraction of phenolic compounds from the olive leaf. The highest total yield using PLE, with ethanol:water, 10.3 MPa, $60{ }^{\circ} \mathrm{C}$ and 110 min was of $30.91 \%$. The PLE 
extract obtained with ethanol at $60{ }^{\circ} \mathrm{C}$ presented the highest concentration of oleuropein (73.65 mg. $\mathrm{g}^{-1}$ extract).

\section{Microwave-assisted extraction}

In microwave-assisted extraction (MAE), the microwave energy heats the solvents that are in contact with solid samples in order to allow the compounds of interest to be shared (Luque De Castro, 2004). This technique fulfills the requirements of green chemistry, because it uses less solvent, has a low cost of extraction with an increase in production and, as a main advantage, it reduces the time of extraction. The reduced extraction time is attributed to the difference between conventional heating and microwave heating (Wang et al., 2005). Three steps are involved in this type of extraction: the separation of solutes present in the active sites of plant matrices by increasing temperature and pressure; diffusion of the solvent through the solid sample; and the release of the solutes into the solvent (Alupului et al., 2012).

The extraction of oleuropein from olive leaves using the microwave method was described by da Rosa et al. (2019) three different extraction techniques were used and compared to the extract of phenolic compounds from olive leaves using a mixture of water or ethanol and different temperatures. MAE at a higher temperature $\left(86^{\circ} \mathrm{C}\right)$ was more efficient in terms of TP yield, with short extraction time (3 min). MAE performed with water as a solvent was effective in disrupting the olive leaf cells thereby promoting the release of the compounds. Under this condition, the TP yield was increased by $82 \%$ when compared to maceration.

Japón-Luján et al. (2006) used microwave-assisted extraction in olive leaves with a mixture of ethanol and water $(80: 20)$, and obtained $2.3 \%$ of oleuropein in the extract.

Sahin et al. (2017) used microwave-assisted extraction without solvents and obtained a maximum yield of oleuropein of $0.060 \mathrm{ppm}$ in leaves of $O$. europea, with the following optimized conditions: $250 \mathrm{~W}$ of irradiation power, 2 min extraction time and $5 \mathrm{~g}$ amount of sample. Water was used as pre-treatment to improve the extraction process.

\section{Microfluidic system (microchannels)}

Microchannel devices are currently widely used for the most varied applications in the food industry. In these systems, lower molecular distance leads to greater diffusion, increasing the possibility of mass transfer (Hisamoto et al., 2001). One of the major differences between microchannels and large-scale devices is the increased surface / volume ratio. Accordingly, miniaturization can be an effective way of increasing heat and mass transfer rate. This type of process can reduce the volume of solvents, the process time and the cost of mass production (Faryadi et al., 2014; Wang et al., 2005)

In a study with the use of microfluidic devices for the extraction of oleuropein, using ethyl acetate in aqueous phase, Naleini et al. (2015) obtained a $68.7 \%$ yield of oleuropein. This was measured in relation to the content in the feed extract of the microfluidic device under optimized aqueous phase $\mathrm{pH}$ conditions, temperature, flow rate and residence time. In this research, the microchannel method was compared to a conventional method, and the authors observed higher yield in extraction, simplicity of operation, and an economical and environmentally friendly procedure, all of which were undoubted advantages.

Yasemi et al. (2017) evaluated the efficiency of the method of microchannels for extracting oleuropein, were obtained a yield of $96.29 \%$ of oleuropein using this system with optimized operating parameters; this value was much higher than the yields found through maceration, Soxhlet and ultrasound-assisted methods.

Heydarid et al., (2018) studied the optimization of oleuropein extraction from organic extracts using a microfluidic device and response surface methodology. The maximum extraction yield was obtained under the following conditions: deionized water as extractant phase, temperature of $40^{\circ} \mathrm{C}$, flow rate ratio of 0.16 and residence time of $0.1010 \mathrm{~min}$. The extraction yield of $70.93 \%$ was obtained under the above conditions with relative standard deviation of $2.0 \%$.

\section{Extraction with membranes}

In recent years, membrane technologies for the separation, purification and concentration of bioactive compounds from aqueous solutions have gained great attention and have showed an increasing potential for application (Khemakhem et al., 2017; Ranieri et al., 2018; Piacentini et al., 2019). This technique is based on the principle of selective permeation of solute molecules by means of a semipermeable polymer or inorganic support, namely, membranes. These structures provide a high quality of phenolic compound concentration due to low operating temperature, besides providing low energy consumption (Cassano et al., 2013; Kim et al., 2015).

Khemakhem et al. (2017) obtained an extract rich in oleuropein (1685 mg. $100 \mathrm{~g}^{-1}$ extract in the retained nanofiltration material) from olive leaves combining microfiltration, ultrafiltration and nanofiltration. In this study, a water extract was prepared and then subjected to molecular size-based sorting. Microfiltration (0.2 ìm) was initially performed to remove large particles, followed by ultrafiltration to remove molecules larger than $5 \mathrm{kDa}$ and, at the end nanofiltration (300 Da), to provide the concentration of polyphenols, mainly oleuropein. 


\section{Others methods}

Differents oleuropein extraction techniques are emerging due to the need to obtain and recover this compounds that are present in residues from the production of olives. Optimization of infrared-assisted extraction was conducted by Abi-Khattar et al., (2019) using Response Surface Methodology (RSM) in order to intensify polyphenol recovery from olive leaves. The extraction efficiency using Ired-Irrad ${ }^{\circledR}$, a newly-patented infrared apparatus (IR), was compared to water bath (WB) conventional extraction. Under optimal conditions, as suggested by the model and confirmed experimentally, the total phenolic content yield was enhanced by more than $30 \%$ using IR as contrasted to $\mathrm{WB}$, which even required $27 \%$ more ethanol consumption. High performance liquid chromatography analyses quantified the oleuropein in $18 \%$.

Lamprou et al. (2020) studied, a new cost effective process for the extraction of phenolic compounds from olive leaves was examined by acid hydrolysis $\left(\mathrm{H}_{2} \mathrm{SO}_{4}\right)$. The resultant extract, after hydrolysis, contained an optimum amount of oleuropein of $43.2 \mathrm{mg} \cdot \mathrm{g}^{-1}$ (dry weight basis).

A plausible alternative to conventional extractants to be used in liquid-liquid extraction us ionic liquids (ILs). These are called "green solvents," because of their physicochemical properties, ease of recovery, and adaptation to specific applications by cation and anion selection. Imidazolium-based ILs are the most studied, because they have a remarkable ability to extract both polar and nonpolar compounds (Visser et al., 2003). They also have a wide range of molecules that can be solubilized, given their ability to change their degree of hydrophobicity. In addition, they have high conductivity and low viscosities (Belhocine et al., 2011) belongs to this class of ILs. On the other hand, phosphonium-based ILs, have high chemical and thermal stability at temperatures under $250^{\circ} \mathrm{C}$.

Julio et al. (2019) proposed to use liquid-liquid extraction to obtain oleuropein from model solutions. Considering the organic phase, two conventional solvents, namely, n-butyl acetate and ethyl acetate and three ionic liquids, were used. The effects of stirring time on the extraction percentages $(\% \mathrm{E})$ of oleuropein, together with variations of distribution coefficient (Dc) with different aqueous-organic (A/O) volumetric ratios, were analyzed. A mathematical model to calculate the liquidliquid extraction was presented. Experimental results showed that $[\mathrm{P} 6,6,6,14][\mathrm{DCA}]$ was the solvent with the highest extraction efficiency.

The content of these leaves varies according to many factors such as climatic conditions, moisture content, age and variety of the plant, agricultural practices, and the extraction procedures used. Thus, extraction is an important and determinative step in the analysis and the use of the cellular bioactive compounds contained in these leaves.Therefore, the identification of the appropriate extraction methods is a limiting step to increase the yield of these compounds and to provide a more efficient purification (Ghomari et al., 2019).

The extraction of phenolic compounds, such as oleuropein, influenced by several experimental parameters depending on these techniques, such as time, temperature and solvent, and with a view to a suitable choice among them, the table 1 shows the advantages and disadvantages of applying each technique.

\section{Methods of purification of oleuropein}

The technique usually applied for the purification of extracts with oleuropein is column chromatography. This process occurs by the separation of two phases (solid and liquid), based on the adsorption capacity and solubility, where the dynamic equilibrium is established between the concentration of the solute in the two phases. The target compound is eluted in a stationary phase with a mixture of suitable solvents. This type of chromatography is simple, efficient and inexpensive. At the end of the purification the obtained fractions must be collected according to their chromatographic profile (Skoog, 1992).

Column chromatography on silica gel using a mixture of methanol and ethyl acetate $(1: 13, \mathrm{v} / \mathrm{v})$ as eluent was applied by Zun-Qiu et al. (2015) to separate and obtain pure oleuropein from olive leaves. In this study, $13.52 \%$ of oleuropein was obtained with a purity of $96.54 \%$, and purification efficiency of $78.49 \%$.

Dang, Nie \& Liang (2010) purified crude extracts of oleuropein by column chromatography, using as the stationary phase sephadex LH-20 and as the mobile phase $50 \%$ ethanol. The purity of oleuropein obtained was $82.9 \%$. Flavonoids and oleuropein were selectively purified from leaves and O. europaea with LSA-21 resin by Li et al. (2011), in a study which evaluated the performance and separability of eight macroporous resins (D101, DM130, HPD450, LSA -21, LSA-40, 07C, LSD001 and HPD600). In this study, LSA-21 resin had better adsorption properties, and the content of total flavonoids and oleuropein in the final purified products increased 13, 2 and 7.5-fold in $87.9 \%$ and $85.6 \%$ recovery yields, respectively.

Andreadou et al. (2006) purified extracts of olive leaves to isolate oleuropein by column chromatography, using as the mobile phase a solution composed of $\mathrm{CH}_{2} \mathrm{Cl}_{2}$ : methanol (98: 2), and Si 60 Merck (15-40 $\mathrm{mm}$ ) as the stationary phase. Purified oleuropein was obtained in glycosidic form, free of aglycone forms, and with purity of $95 \%$. 


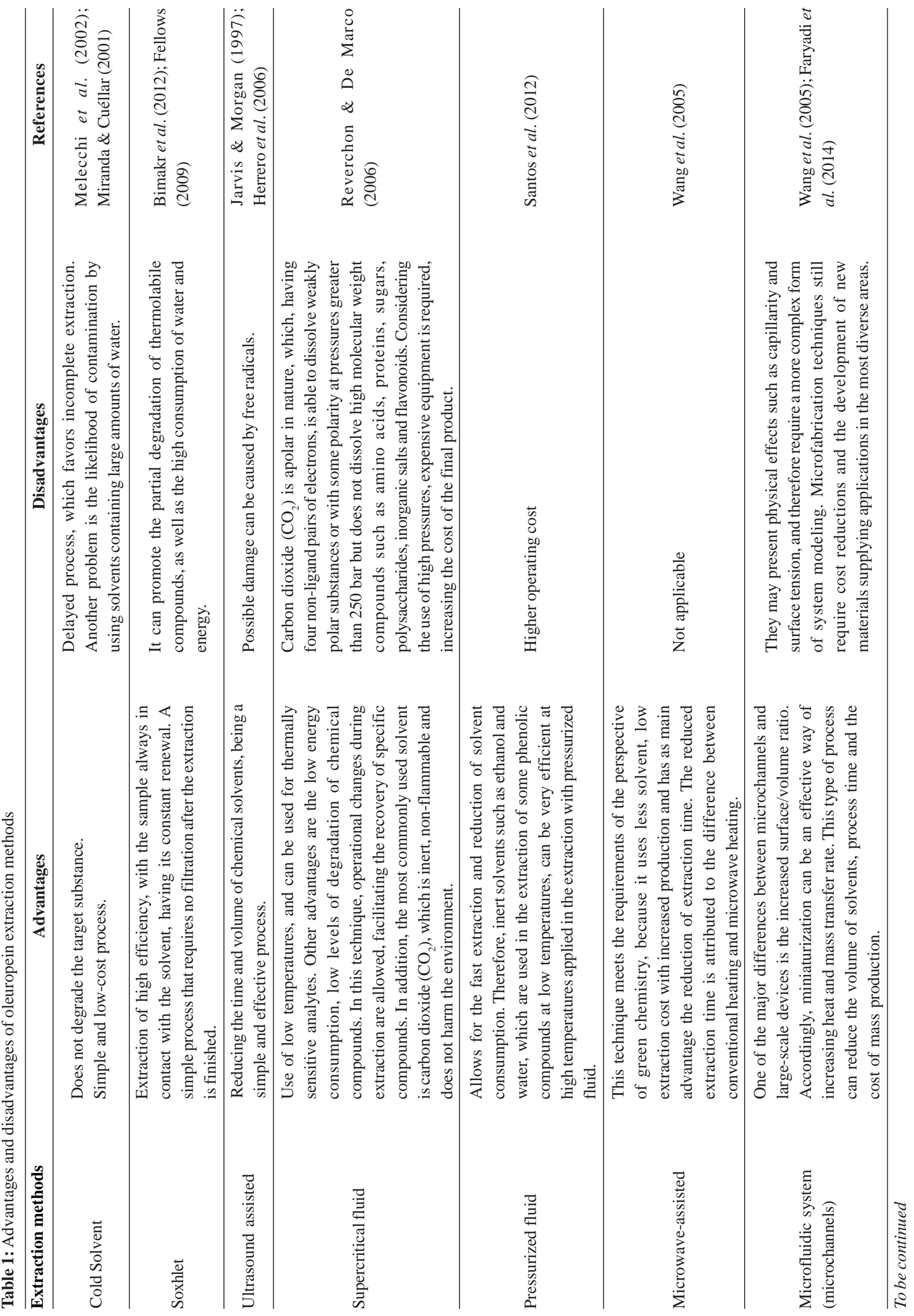

Rev. Ceres, Viçosa, v. 67, n.4, p. 315-329, jul/aug, 2020 
The adsorption process in the macroporous resins is reversible owing to the difference in the polarity, pore size, and specific surface area of the resins; the adsorption and desorption of the effective components vary. In practical applications, the resin is required to adsorb large amounts of the target components, and the desorption rate is required to be high to ensure the maximum recovery of the effective components. Therefore, Li et al. (2019) study simultaneous purification and separation of oleuropein, were 11 macroporous resins were preliminarily screened by static adsorption and desorption experiments. By comparing the ability of 11 macroporous resins to adsorb and desorb oleuropein, HPD-100B was selected as the ideal resin for the separation and enrichment of the component. The static adsorption equilibria of oleuropein on the HPD-100B resin were well fitted to the Langmuir isotherm model at various temperatures. Optimal separation conditions were achieved by the following kinetic and dynamic adsorption/desorption experiments: $60 \mathrm{BV}$ loading amount of adsorption at $25^{\circ} \mathrm{C}$ with a flow rate of $3 \mathrm{BV} / \mathrm{h}$, followed by desorption with a gradient elution of $20 \%$ ethanol (7 BV) and $40 \%$ ethanol (9 BV) at $25^{\circ} \mathrm{C}$ and $3 \mathrm{BV} / \mathrm{h}$. The purity of oleuropein obtained under the optimized conditions increased by 8.2-fold compared with that of the initial extract. The recovery of oleuropein were $89.8 \%$. This method demonstrates a practical approach for bulk extraction and purification of oleuropein in an economical way.

Khemakhem et al. (2017) studied the combination of different technologies such as micro, ultra and nanofiltration for separation and concentration of oleuropein. They concluded that the separation of compounds by membrane constitutes an attractive alternative to conventional processes such as by chromatographic column, showing that these technologies offer unique separation capacity, possibilities for scale expansion and low energy consumption. The authors even concentrated the oleuropein content up to 50 times, reaching an extract

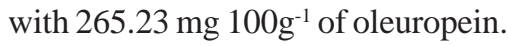

\section{Applications of oleuropein}

A number of studies have been carried out with leaf extracts and olive fruit for technological and functional purposes, but few have been developed with pure oleuropein alone. There are reports of the use of this compound in food preservation as a natural antioxidant (Dua et al., 2015; Al-Rimawi et al., 2017), in sanitizing formulations (Dominciano et al., 2016) and as a functional component in dairy products (Zoidou et al., 2017). The effects of the enzyme-linked immunosorbent assay on the treatment of diseases (Esmailidehaj et al., 2016; Shi et al., 2017) have also been investigated. In addition, oleuropein 
has the potential for use in cosmetics (Perugini et al., 2008). Other studies have demonstrated the antimicrobial properties of oleuropein (Bisignano et al., 1999; Furneri et al., 2002). Figure 4 summarizes the main applications of oleuropein as a coadjuvant in technological and therapeutic processes.

\section{Food preservation and antioxidant potential}

Oxidative processes in foods cause deterioration and consequently induce loss of quality. In a study on the application of oleuropein extracts, an improvement in the lipid oxidative stability and storage quality of Tabaq-Maz (fried ribs popular in India) was observed (Dua et al., 2015). Also, protective effects of oleuropein were observed against lipid oxidation in hamburgers stored at $4^{\circ} \mathrm{C}$, suggesting the commercial use of oleuropein as a natural preservative in meat foods (Al-Rimawi et al., 2017). The antioxidant activity was higher than the original extract obtained from olive leaves (Khemakhem et al., 2017), demonstrating the potential of oleuropein to prevent oxidation of food. Zun-Qiu et al. (2015) extracted and purified oleuropein from olive leaves, and found that oleuropein is more effective than butyl hydroxy toluene (BHT) for application in food and human health, after a comprehensive assessment of antioxidant activity in vitro. A high oleuropein extract added to extra-virgin olive oils and refined olive oil improved the oxidative stability with an increased induction period, which is the period necessary for the products formed in the oxidation to be detected (Coppa et al., 2017).

\section{Functional component in dairy products}

Oleuropein is a bioactive with beneficial properties for human health, because it presents antioxidant, antimicrobial, hypoglycemic and anti-inflammatory activities, among others (Fernández-Bolaños et al., 2006; Guinda, 2006).

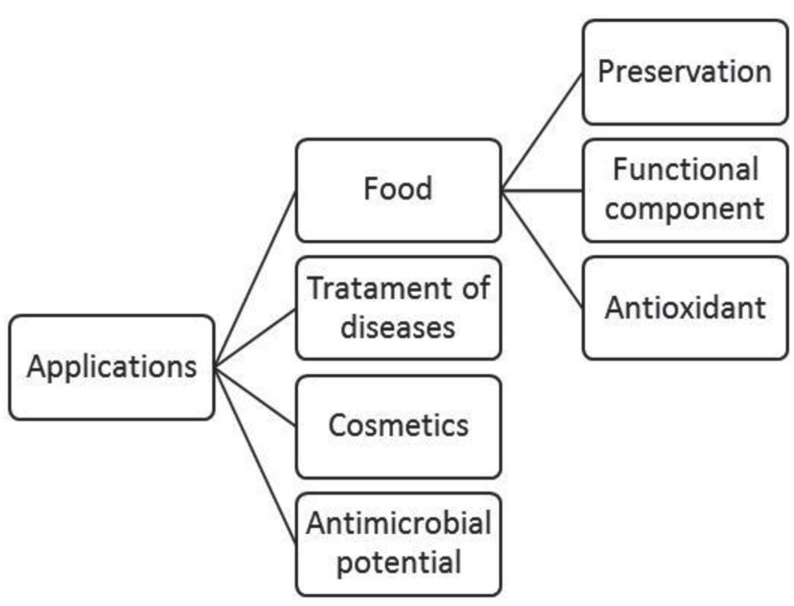

Figure 4: Main applications of oleuropein.
As milk and yogurt are good matrices for the development of various functional foods and popular dairy products with high nutritional value, Dominciano et al. (2016) added oleuropein to these foods to increase health benefits upon ingestion. In this study, the researchers reported that oleuropein was stable at all stages of preparation and during the period of validity of both products, without degradation occurring during the heat treatment of the milk and without hydrolysis by the acids produced in the fermentation process of yogurt. In addition, oleuropein was not metabolized by lactic acid bacteria, and it did not inhibit the growth of these microorganisms. Furthermore, it remained stable in the final products and did not alter the sensorial characteristics of the products. In a study by Zoidou et al. (2017), milk and yogurt added with oleuropein exhibited acceptable taste, color and texture, similar to conventional. In this study, oleurpein was stable during heat treatment, fermentation and storage.

\section{Treatment of diseases}

Several studies have reported positive medicinal effects of the use of oleuropein, as it can act against oxidative stress (Esmailidehaj et al., 2016; Hadrich et al., 2016; Shi et al., 2017; Sun et al., 2017; Fki et al., 2020) in the reduction of body weight, total cholesterol and triglycerides (Andreadou, 2006; Hadrich et al., 2016) as a possible preventive component of diabetes (Wainstein et al., 2012; Del Ben et al., 2019) in combating hypertension (Sun et al., 2017) in the treatment of interstitial hemorrhage (CVA), hepatitis B and cancer (Seçme et al., 2016; Shi et al., 2017; Zhao et al., 2009; Barzegar et al., 2019; Nassir et al., 2019; Zhang \& Zhang 2019), in nonalcoholic fatty liver disease (Santini et al., 2020) in protecting against Alzheimer's, Parkinson's and hemorrhagic cystitis (Sherif et al., 2016) as a preventive/palliative treatment of lupus nephritis (Castejon et al., 2019) and in preventing myocarditis (Grossi et al., 2013; Pasban-Aliabadi et al., 2013). A relevant aspect is that much of the ingested oleuropein is absorbed into the human organism (Vissers et al., 2002).

\section{Cosmetics}

In a study by Perugini et al. (2008), an emulsion and an emulsifier containing oleuropein were prepared to evaluate their cosmetic properties against UVB (Ultra Violet Radiation)-induced erythema. These materials were applied in volunteers before exposure to UVB irradiation to investigate their protective activity, and after UVBinduced erythema, for the analysis of the lenitive effect. The results demonstrated that formulations containing oleuropein exhibit lenitive efficacy with reduced damage caused by UV. 


\section{Antimicrobial potential}

Polystyrene and stainless steel are widely used in food industry equipment and utensils. It is important to study the formation of biofilm on the surface of these materials, since they are difficult to sterilize and failures can lead to contamination in food (Dominciano et al., 2016).

Oleuropein presented a higher antimicrobial effect for multispecies biofilms formed on surfaces of polystyrene microplates, with greater reduction in the association of Listeria monocytogenes, Escherichia coli and Staphylococcus aureus (91 and 49\%, respectively). It increased the efficiency of peracetic acid (APA), a commercial sanitizer, in the inactivation of Listeria monocytogenes biofilms on stainless steel surfaces (Dominciano et al., 2016).

Oleuropein, in concentrations of 20 to $320 \mathrm{mg}$. L-1, showed an inhibitory effect on the growth of Mycoplasma hominis, Mycoplasma fermentans, Mycoplasma pneumoniae and Mycoplasma pirum, in a study by Furneri et al. (2002).

In a study to verify the in vitro antimicrobial activity of oleuropein against pathogenic bacteria in humans, Bisignano et al. (1999) observed that oleuropein inhibited the growth of Salmonella typhi, Vibrio parahaemolyticus and $S$. aureus, with MIC values between 62.5 and $500 \mathrm{ìg}$. mL-1 for ATCC strains and between 31.25 and 250 ìg.mL ${ }^{1}$ for clinical isolates. For Haemophilus influenzae and Moraxella catarrhalis, oleuropein was ineffective at the concentrations tested ( 0.015 to 500 ìg. $\left.\mathrm{mL}^{-1}\right)$.

In the supplementation of olive leaves in the diet of chickens, after slaughtering the thighs and drumsticks of the chickens that received olive leaves showed greater microbiological stability than the control, in which $5 \mathrm{~g} / \mathrm{kg}$ prevented the growth of Staphylococcus aureus, psychrotrophic and mesophilic aerobes and $10 \mathrm{~g} / \mathrm{kg}$ prevented the growth of Enterococcus spp., lactic acid bacteria, thermotolerant and total coliforms, Pseudomonas, Clostridium perfringens and Escherichia coli (p <0.05). The results indicate the feasibility of using olive leaves as a food supplement, improving the microbiological quality of chicken meat. The advantages of using olive leaves were granted due to the presence of polyphenols and oleuropein (Marangoni et al., 2015).

\section{CONCLUSIONS}

Oleuropein is a bitter glycoside, present in all parts of the olive tree, but mainly in the leaves, and studies indicate that this phenolic compound has important antiinflammatory, antimicrobial and antiviral activities, among others. Its ability to act as a natural antioxidant has also attracted the attention of researchers, as consumers increasingly seek natural products or those that contain natural constituents in their formulation, prompting the food and cosmetics industry to consider replacing synthetic antioxidants with those extracted from vegetable sources. This phenol can be extracted by techniques involving the use of cold solvents, Soxhlet, ultrasound, supercritical fluids, pressurized fluid, microwave, microfluidic system and filtration. Extraction of phenolic compounds such as oleuropein is influenced by several experimental parameters depending on these techniques, such as time, temperature and type of solvent. Studies aiming at obtaining this compound have been conducted, seeking the optimization of the process as well as the search for "clean" technologies that use non-toxic solvents and present a low cost.

\section{REFERENCES}

Abi-Khattar AM, Rajha HN, Abdel-Massih RM, Maroun RG, Louka N \& Debs E (2019) Intensification of polyphenol extraction from olive leaves using Ired-Irrad $囚$, an environmentally-friendly innovative technology. Antioxidants, 8:227.

Ahamad J, Toufeeq I, Khan MA, Ameen MSM, Anwer ET, Uthirapathy S \& Ahmad J (2019) Oleuropein: A natural antioxidant molecule in the treatment of metabolic syndrome. Phytotherapy Research, 33:3112-3128.

Al-Rimawi F \& Tarawa MS \& Elama C (2017) Olive leaf extract as natural antioxidant additive of fresh hamburger stored at $4^{\circ} \mathrm{C}$. American Journal of Food Science and Technology, 5:162166.

Alupului A, Calinescu I \& Lavric V (2012) Microwave extraction of active principles from medicinal plants. UPB Science Bulletin, 74:1454-2331.

Andreadou I (2006) The olive constituent oleuropein exhibits anti-ischemic, antioxidative, and hypolipidemic effects in anesthetized rabbits. The journal of nutrition, 136:2213-2219.

Baldino L, Della Porta G, Osseo LS, Reverchon E \& Adami R (2018) Concentrated oleuropein powder from olive leaves using alcoholic extraction and supercritical CO2 assisted extraction. The Journal of Supercritical Fluids, 133:65-69.

Barzegar F, Zaefizadeh M, Yari R \& Salehzadeh A (2019 A. Synthesis of Nano-Paramagnetic Oleuropein to Induce KRAS OverExpression: A New Mechanism to Inhibit AGS Cancer Cells. Medicina, 55:388-400.

Bastante CC, Cardoso LC, Ponce MF, Serrano CM \& De La OssaFernández EM (2018) Characterization of olive leaf extract polyphenols loaded by supercritical solvent impregnation into PET/PP food packaging films. The Journal of Supercritical Fluids, 140:196-206.

Bayraktar O, Köse MD \& Baspinar Y (2019) Development of olive leaf extract loaded fibroin microparticles by spray drying. The Open Drug Discovery Journal, 13:39-45.

Bayram M, Topuz S \& Kaya C (2020) Antioxidant, Antimicrobial Activity of Olive Leaf Extract and Oleuropein, Their Possibilities Usage in Foods. Turkish Journal of Agriculture-Food Science and Technology, 8:337-347.

Belhocine T, Forsyth SA, Gunaratne HQN, Nieuwenhuyzen M, Puga AV, Seddon KR, Srinivasan G \& Whiston K (2011) New ionic liquids from azepane and 3-methylpiperidine exhibiting wide electrochemical windows. Green Chemistry, $13: 59-63$. 
Bendicho C, De La Calle I, Pena F, Costas M, Cabaleiro N \& Lavilla I (2012) Ultrasound-assisted pretreatment of solid samples in the context of green analytical chemistry. TrAC Trends Analytical Chemistry, 31:50-60.

Benincasa C, Santoro I, Nardi M, Cassano A \& Sindona G (2019) Eco-Friendly Extraction and Characterisation of Nutraceuticals from Olive Leaves. Molecules, 24:3481-3495.

Böhmer-Maas BW, Otero DM \& Zambiazi RC (2020) Optimization of the extraction of phenolic compounds from olive pomace using response surface methodology. Revista Ceres, 67:164170

Bimakr M, Rahman A, Taip S, Adzahan M, Sarker M, Islam Z \& Ganjloo A (2012) Optimization of ultrasound-assisted extraction of crude oil from winter melon (Benincasa hispida) seed using response surface methodology and evaluation of its antioxidant activity, total phenolic content and fatty acid composition. Molecules, 17:11748-11762.

Bisignano G, Tomaino A, Cascio L, Crisafi G, Uccella N \& Saija A (1999) On the in vitro antimicrobial activity of oleuropein and hidroxitirosol. Journal of Pharmacy and Pharmacology, 51:971974

Bonechi C, Donati A, Tamasi G, Pardini A, Rostom H, Leone G \& Rossi C (2019) Chemical characterization of liposomes containing nutraceutical compounds: Tyrosol, hydroxytyrosol and oleuropein. Biophysical chemistry, 246:25-34.

Bonoli M, Bendini A, Cerretani L, Lercker G \& Toschi G (2004) Qualitative and semiquantitative analysis of phenolic compounds in extra virgin olive oils as a funtion of the ripening degree of olive fruits by different analytical techniques. Journal of Agricultural and Food Chemistry, 52:7026-7032.

Cassano A, Conidi C, Giorno L \& Drioli E (2013) Fractionation of olive mill wastewaters by membrane separation techniques. Journal of hazardous materials, 248:185-193.

Castejon ML, Sánchez-Hidalgo M, Aparicio-Soto M, Montoya T \& Alarcón-de-la-Lastra C (2019) Dietary oleuropein and its new acyl- derivate attenuate murine lupus nephritis through HO-1/Nrf2 activation and suppressing JAK/STAT, NF-kB MAPK and NLRP3 inflammasome signaling pathways. The Journal of Nutritional Biochemistry, 74:108229.

Cavaca LA, López-Coca IM, Silvero G \& Afonso CA (2020) The olive-tree leaves as a source of high-added value molecules: Oleuropein. In: Rahman AU (Ed.) Studies in Natural Products Chemistry. Netherlands, Elsevier. p.131-180.

Cavaca LA, López-Coca IM, Silvero G \& Afonso CA (2020) The olive-tree leaves as a source of high-added value molecules: Oleuropein. Studies in Natural Products Chemistry, 64:131-180.

Charpe W \& Rathod K (2012) Extraction of glycyrrhizic acid from licorice root using ultrasound: Process intensification studies. Chemical Engineering and Processing: Process Intensification, 54:37-41

Cho WY, Kim DH, Lee HJ, Yeon SJ \& Lee CH (2020) Journal of Food Quality Evaluation of Effect of Extraction Solvent on Selected Properties of Olive Leaf Extract. Journal of Food Quality, 2020: 3013649.

Chung H, Ji X, Canning C, Sun S \& Zhou K (2010) Comparison of different strategies for soybean antioxidant extraction. Journal of Agricultural and Food Chemistry, 58:4508-4512.

Cifá D, Skrt M, Pittia P, Di Mattia C \& Poklar Ulrih N (2018) Enhanced yield of oleuropein from olive leaves using ultrasound assisted extraction. Food Science \& Nutrition, 6:1128-1137.

Coppa C, Rosim E, Oliveira F, Rodrigues C \& Gonçalves B (2017) Extraction of oleuropein from olive leaves using a hydroalcoholic solvent. Brazilian Journal of Food Technology, 20:89-93.
Damtoft S, Franzyk H \& Jensen R (1992) Excelsioside, a secoiridoid glucoside from Fraxinus excelsior. Phytochemistry, 31:41974201.

Dang J, Nie X \& Liang L (2010) Purification of oleuropein by sephadex LH-20 column. Journal of Chinese medicinal material, 33:119-121.

Del Ben M, Nocella C, Loffredo L, Bartimoccia S, Cammisotto V, Mancinella M, Angelico F, Valenti V, Cavarretta E, Carnevale E \& Violi F (2019) Oleuropein-enriched chocolate by extra virgin olive oil blunts hyperglycaemia in diabetic patients: Results from a one-time 2-hour post-prandial cross over study. Clinical Nutrition, 39:2187-2191.

Del Mar-Contreras M, Lama-Muñoz A, Espínola F, Moya M, Romero I \& Castro E (2020) Valorization of olive mill leaves through ultrasound-assisted extraction. Food Chemistry, $314: 126218$.

Dominciano LCC, Oliveira CAF, Lee SH \& Corassin CH (2016) Individual and combined antimicrobial activity of oleuropein and chemical sanitizers. Journal of Food Chemistry and Nanotechnology, 2:124-127.

Dua S, Bhat F \& Kumar S (2015) Effect of oleuropein on the oxidative stability and storage quality of Tabaq-Maz, fried mutton ribs. Food Bioscience, 12:84-92

Duarte C (2011) Extração e encapsulamento de compostos bioactivos do bagaço de azeitona. Dissertação de Mestrado. Universidade Técnica de Lisboa, Lisboa. 113p.

Esmailidehaj M, Bajoovand S, Rezsvani E, Sherifidehaj M \& Hafezimoghadam Z (2016) Effect of oleuropein on myocardial dysfunction and oxidative stress induced by ischemic-reperfusion injury in isolated rat heart. Journal of Ayurveda and Integrative Medicine, 7:224-230.

Faryadi M, Rahimi M, Safari S \& Moradi N (2014) Effect of high frequency ultrasound on micromixing efficiency in microchannels. Chemical Engineering and Processing: Process Intensification, 77:13-21.

Fellows J (2009) Food processing technology: Principles and Practice. $3^{\mathrm{a}}$ ed. Cambridge, Woodhead Publishing Ltda. 912p.

Fernández-Bolaños J, Rodríguez G, Rodríguez R, Guillén R \& Jiménez A (2006) Potential use of olive by-products, extraction of interesting organic compounds from olive oil waste. Grasas y Aceites, 57:95-106.

Fki I, Sayadi S, Mahmoudi A, Daoued I, Marrekchi R \& Ghorbel H (2020) Comparative Study on Beneficial Effects of Hydroxytyrosol-and Oleuropein-Rich Olive Leaf Extracts on High-Fat Diet-Induced Lipid Metabolism Disturbance and Liver Injury in Rats. BioMed Research International, 2020:01-15.

Furneri PM, Marino A, Saija A, Uccella N \& Bisignano G (2002) In vitro antimycoplasmal activity of oleuropein. International journal of antimicrobial agents, 20:293-296.

Galanakis M, Tornberg E \& Gekas V (2010) Recovery and preservation of phenols from olive waste in ethanolic extracts. Journal of Chemical Technology \& Biotechnology, 85:11481155 .

Ghelichkhani G, Modaresi MH, Rashidi L, Shariatifar N, Homapour M \& Arabameri M (2019) Effect of the spray and freeze dryers on the bioactive compounds of olive leaf aqueous extract by chemometrics of HCA and PCA. Journal of Food Measurement and Characterization, 13:2751-2763.

Ghomari O, Sounni F, Massaoudi Y, Ghanam J, Kaitouni LBD, Merzouki M \& Benlemlih M (2019) Phenolic profile (HPLCUV) of olive leaves according to extraction procedure and assessment of antibacterial activity. Biotechnology Reports, 23:e00347.

Rev. Ceres, Viçosa, v. 67, n.4, p. 315-329, jul/aug, 2020 
Giacometti J, •auhar G \& •uviæ M (2018) Optimization of ultrasonic-assisted extraction of major phenolic compounds from Olive leaves (Olea europaea L.) using response surface methodology. Foods, 7:149.

Grossi C, Rigacci S, Ambrosini S, Dami E, Luccarini I, Traini C \& Stefani M (2013) The polyphenol oleuropeína aglycone protects TgCNRD8 mice against Aâ plaque pathology. Plos One, 8:e71702

Guinda A (2006) Use of solid residue from the olive industry. Grasas Y Aceites, 57:107-115.

Hadrich F, Mahmoudi A, Bouallagui Z, Feki I, Isoda H, Feve B \& Sayadi S (2016) Evaluation of hypocholesterolemic effect of oleuropein in cholesterol-fed rats. Chemico-biological interactions, 25:254-60.

Herrero M, Cifuentes A \& Ibañez E (2006) Sub-and supercritical fluid extraction of funtional ingredientes from different natural sources: plants, food-byproducts, algae and microalgae. Food Chemistry, 98:136-148.

Heydarid R, Rahimi M \& Naleini N (2018) Optimization of Oleuropein Extraction from Organic Extracts Using a Microfluidic Device and Response Surface Methodology. Herbal Medicines Journal, 3:60-69.

Hisamoto H, Horiuchi T, Tokeshi M, Hibara A \& Kitamori T (2001) On-chip integration of neutral ionophore-based ion pair extraction reaction. Analytical Chemistry, 73:1382-1386.

Irakli M, Chatzopoulou P \& Ekateriniadou L (2018) Optimization of ultrasound-assisted extraction of phenolic compounds: Oleuropein, phenolic acids, phenolic alcohols and flavonoids from olive leaves and evaluation of its antioxidant activities. Industrial Crops and Products, 124:382-388.

Japón-Luján R, Luque-Rodríguez M \& De Castro L (2006) Dynamic ultrasound-assisted extraction of oleuropeína and related biophenols from olive leaves. Journal of Chromatographic A, 1108:76-82.

Jarvis P \& Morgan D (1997) Isolation of plant products by supercritical-fluid extraction. In: Jarvis AP \& Morgan ED (Eds.) Phytochemical Analysis: An International Journal of Plant Chemical and Biochemical Techniques, 8:217-222.

Julio R, Zambra C, Merlet G, Cabezas R, Correa G, Salinas G \& Lemus-Mondaca R (2019) Liquid-liquid extraction of hydroxytyrosol, tyrosol, and oleuropein using ionic liquids. Separation Science and Technology, 54:2895-2906.

Jaski JM, Barão CE, Morais Lião L, Da Silva Pinto V, Zanoelo EF \& Cardozo-Filho L (2019) â-Cyclodextrin complexation of extracts of olive leaves obtained by pressurized liquid extraction. Industrial Crops and Products, 129:662-672.

Kabbash EM, Ayoub IM, Abdel-Shakour ZT \& El-Ahmady SH (2019) A Phytochemical Study on Olea europaea L. Olive Leaf Extract (cv. Koroneiki) growing in Egypt. Archives of Pharmaceutical Sciences Ain Shams University, 3:99-105.

Khemakhem I, Gargouri D, Dhouib A, Ayadi A \& Bouaziz M (2017) Oleuropein rich extract from olive leaves by combining microfiltration, ultrafiltration and nanofiltration. Separation and Purification Technology, 172:310-317.

Kim K, Jung Y, Kwon H \& Yang W (2015) Dynamic microfiltration with a perforated disk for effective harvesting of microalgae. Journal of Membrane Science, 475:252-258.

Lama-Muñoz A, Contreras MDM, Espínola F, Moya M, Romero I \& Castro E (2019) Optimization of Oleuropein and Luteolin7-O-Glucoside Extraction from Olive Leaves by UltrasoundAssisted Technology. Energies, 12:2486.
Lamprou GK, Vlysidis A, Tzathas K \& Vlyssides AG (2020) Statistical optimization and kinetic analysis of the extraction of phenolic compounds from olive leaves. Journal of Chemical Technology and Biotechnology, 95:457-465.

Li C, Zheng Y, Wang X, Feng S \& Di D (2011) Simultaneous separation and purification of flavonoids and oleuropein from Olea europaea L. (olive) leaves using macroporous resin. Journal of the Science of Food and Agriculture, 91:2826-34.

Li G, Yan H \& Liu X (2019) Simultaneous Purification and Separation of Syringoside and Oleuropein from Syringa oblata Lindl. Extract Using Macroporous Resin. Journal of Chemistry, 2019:548-558.

Liu Y, Dai W \& Ye S (2019) The olive constituent oleuropein exerts nephritic protective effects on diabetic nephropathy in $\mathrm{db} / \mathrm{db}$ mice. Archives of physiology and biochemistry, DOI: $10.1080 / 13813455.2019 .1691603$.

Luque De Castro D (2004) Supercritical fluid technology for drug product development. New York, Marcel Dekker. 632p.

Marangoni C, Cichoski José A, Barin Smanioto J \& Menezes Ragagnin C (2015) Effect of incorporating olive leaves (Olea europaea L.) on the development and quality of poultrymeat. Brazilian Journal Food Technology, 18:173-184.

Melecchi MIS, Caramão EB, Nascimento Filho I, Abad FC, Zini PP \& Martinez MM (2002) Chemical Composition of Hibiscus tiliaceus L. flowers: A Study of Extraction Methods. Journal of separation Science, 25:86-90.

Miranda M \& Cuéllar A (2001) Farmacognosia y Products Naturales. Havana, Editorial Felipe Varela. 437p.

Mosca M, Cuomo F, Lopez F \& Ceglie A (2013) Role of emulsifier layer, antioxidants and radical initiators in the oxidation of olive oil-in-water emulsions. Food Research International, 50:377-383

Mustafa A \& Turner C (2011) Pressurized liquid extraction as a green approach in food and herbal plants extraction: a review. Analytica Chimica Acta, 703:8-18.

Naleini N, Rahimi M \& Heydari R (2015) Oleuropein extraction using microfluidic system. Chemical Engineering and Processing: Process Intensification, 92:01-06.

Nassir A, Ibrahim I, Md S, Waris M, Ain M, Ahmad I \& Shahzad N (2019) Surface functionalized folate targeted oleuropein nanoliposomes for prostate tumor targeting: in vitro and in vivo activity. Life Science, 220:136-146.

Nediani C, Ruzzolini J, Romani A \& Calorini L (2019) Oleuropein, a Bioactive Compound from Olea europaea L., as a Potential Preventive and Therapeutic Agent in Non-Communicable Diseases. Antioxidants, 8:578.

Omar SH (2010) Oleuropein in olive and its pharmacological effects. Scientia Pharmaceutica, 78:133-154.

Papachristodoulou A, Komianos K, Psarras N, Efentakis P, Varela E, Davos C \& Tsitsilonis O (2019) The natural product Oleuropein shows enhanced anticancer and cardioprotective activity when co-administered in vivo with Doxorubicin. Planta Medica, 85:1552.

Pasban-Aliabadi H, Esmaeili-Mahani S, Sheibani V, Abbasnejad M, Mehdizadeh A \& Yaghoobi M (2013) Inhibition of 6hydroxydopamine-induced PC12 cell apoptosis by olive (Olea europaea L.) leaf extract is performed by its main component oleuropein. Rejuvenation Research, 16:134-142.

Perugini P, Vettor M, Rona C, Troisi L, Villanova L, Genta I, Conti B \& Pavanetto F (2008) Efficacy of oleuropein against UVB irradiation: preliminary evaluation. International Journal of Cosmetic Science, 30:113-120. 
Piacentini E, Mazzei R, Bazzarelli F, Ranieri G, Poerio T \& Giorno L (2019) Oleuropein Aglycone Production and Formulation by Integrated Membrane Process. Industrial and Engineering Chemistry Research, 58:16813-16822.

Przychodzen P, Wyszkowska R, Gorzynik-Debicka M, Kostrzewa T, Kuban-Jankowska A \& Gorska-Ponikowska M (2019) Anticancer potential of oleuropein, the polyphenol of olive oil, with 2-methoxyestradiol, separately or in combination, in human osteosarcoma cells. Anticancer research, 39:1243-1251.

Ramírez E, Brenes M, García P, Medina E \& Romero C (2016) Oleuropein hydrolysis in natural green olives: Importance of the endogenous enzymes. Food Chemistry, 206:204-209.

Ranieri G, Mazzei R, Poerio T, Bazzarelli F, Wu Z, Li K \& Giorno L (2018) Biorefinery of olive leaves to produce dry oleuropein aglycone: Use of homemade ceramic capillary biocatalytic membranes in a multiphase system. Chemical Engineering Science, 185:149-156.

Reverchon E \& De Marco I (2006) Supercritical fluid extraction and fractionation of natural matter. The Journal of Supercritical Fluids, 38:146-166.

Romero C, Medina E, Mateo A \& Brenes M (2017) Quantification of bioactive compounds in Picual and Arbequina olive leaves and fruit. Journal of the Science of Food and Agriculture, 97:1725-1732.

Romero C, García P \& Brenes M (2020) Chemical hydrolysis of oleuropein affected by the type of organic acid. Food Chemistry, 316:126351-126357.

Rosa GS, Vanga S, Gariepy Y \& Raghavan V (2019) Comparison of microwave, ultrasonic and conventional techniques for extraction of bioactive compounds from olive leaves (Olea europaea L.). Innovative Food Science \& Emerging Technologies, 58:102234.

aahin S, Samli R, Tan B, Barba J, Chemat F, Cravotto G \& Lorenzo M (2017) Solvent-free microwave-assisted extraction of polyphenols from olive tree leaves: Antioxidant and antimicrobial properties. Molecules, 22:1056-1069.

Santini SJ, Porcu C, Tarantino G, Amicarelli F \& Balsano C (2020) Oleuropein overrides liver damage in steatotic mice. Journal of Functional Foods, 65:103756.

Santos T, Veggi C \& Meireles A (2012) Optimization and economic evaluation of pressurized liquid extraction of phenolic compounds from jabuticaba skins. Journal of Food Engineering, 108:444-452.

Seçme M, Eroðlu C, Dodurga Y \& Baðcý G (2016) Investigation of anticancer mechanism of oleuropein via cell cycle and apoptotic pathways in SH-SY5Y neuroblastoma cells. Gene, 585:93-99.

Sherif O, Nakshabandi M, Mohamed A \& Sarhan M (2016) Uroprotective effect of oleuropein in a rat model of hemorrhagic cystitis. The International Journal of Biochemistry \& Cell Biology, 74:12-17.

Shi J, Wu G, Zou X \& Jiang K (2017) Oleuropein protects intracerebral hemorrhage-induced disruption of blood-brain barrier through alleviation of oxidative stress. Pharmacological Reports, 69:1206-1212.

Shirzad H, Niknam V, Taheri M \& Ebrahimzadeh H (2017) Ultrasound-assisted extraction process of phenolic antioxidants from Olive leaves: a nutraceutical study using RSM and LCESI-DAD-MS. Journal of food science and technology, 54:23612371.

Sivakumar G, Uccella NA \& Gentile L (2018) Probing downstream olive biophenol secoiridoids. International Journal of Molecular Sciences, 19:2892
Skoog A (1992) Principles of Instrumental Analysis. 4 ed. Fort Worth, Saunders College Pub. 700p.

Soria C \& Villamiel M (2010) Effect of ultrasound on the technological proprieties and bioactivity of food: a review. Trends in Food Science and Technology, 21:323-331.

Sucharitha P, Satyanarayana SV \& Reddy KB (2019) Pretreatment and optimization of processing conditions for extraction of oleuropein from olive leaves using central composite design. Pharmacognosy Research, 11:178-187.

Sun W, Wang X, Hou C, Yang L, Li H, Guo J \& Kang Y (2017) Oleuropein improves mitochondrial function to attenuate oxidative stress by activating the Nrf2 pathway in the hypothalamic paraventricular nucleus of spontaneously hypertensive rats. Neuropharmacology,13:556-566.

Taylor T (1996) Supercritical fluid extraction. New York, John Wiley \& Sons Inc. 182p.

Tayoub G, Sulaiman H, Hassan AH \& Alorfi M (2012) Determination of oleuropein in leaves and fruits of some Syrian olive varieties. International Journal of Medicinal and Aromatic Plants, 2:428-433.

Toma M, Vinatoru M, Paniwnyk L \& Mason J (2001) Investigation of the effects of ultrasound on vegetal tissues during solvent extraction. Ultrasonics Sonochemistry, 8:137-142.

Tripoli E, Giammanco M, Tabacchi G, Di Majo D, Giammanco S \& La Guardia M (2005) The phenolic compounds of olive oil: structure, biological activity and beneficial effects on human health. Nutrition research reviews, 18:98-112.

Ucella N (2001) Olive biophenols: novel ethnic and technological approach. Trends in Food Science \& Technology, 11:328-339.

Visser AE, Jensen MP, Laszak I, Nash KL, Choppin GR \& Rogers RD (2003) Uranyl coordination environment in hydrophobic ionic liquids: an in situ investigation. Inorganic Chemistry, 42:2197.

Vissers N, Zock L, Roodenburg J, Leenen R \& Katan B (2002) Olive oil phenols are absorbed in humans. The Journal of Nutrition, 132:409-417.

Wainstein J, Ganz T, Boaz M, Bar DY, Dolev E \& Kerem Z (2012) Olive leaf extract as a hypoglycemic agent in both human diabetic subjects and in rats. Journal of Medicinal Food, 15:605-610.

Wang X, Saridara C \& Mitra S (2005) Microfluidic supported liquid membrane extraction. Analytica Chimica Acta, 543:9298.

Xynos N, Papaefstathiou G, Gikas E, Argyropoulou A, Aligiannis N \& Skaltsounis L (2014) Design optimization study of the extraction of olive leaves performed with pressurized liquid extraction using response surface methodology. Separation and Purification Technology, 122:323-330.

Xynos N, Papaefstathiou G, Psychis M, Argyropoulou A, Aligiannis N \& Skaltsounis L (2012) Development of a green extraction procedure with super subcritical fluids to procedure extracts enriched in oleurpein from olive leaves. Journal of Supercritical Fluids, 67:89-93.

Yasemi M, Heydarinasab A, Rahimi M \& Ardjmand M (2017) Microchannels Effective Method for the Extraction of Oleuropein Compared with Conventional Methods. Journal of Chemical, 2017:6594156.

Yateem H, Afaneh I \& Al-Rimawi F (2014) Optimum conditions for oleuropein extraction from olive leaves. International Journal of Applied Science and Technology, 4:153-157. 
Zhang F \& Zhang M (2019) Oleuropein inhibits esophageal cancer through hypoxic suppression of BTG3 mRNA. Food \& Function, 10:978-985.

Zhao G, Yin Z \& Dong J (2009) Antiviral efficacy against hepatitis $B$ virus replication of oleuropein isolated from Jasminum officinale L. var. grandiflorum. Journal of Ethnopharmacology, $125: 265-268$.
Zoidou E, Melliou E, Moatsou G \& Magiatis K (2017) Preparation of Functional Yogurt Enriched With Olive-Derived Products. In: Shah N (Ed.) Yogurt in Health and Disease Prevention. London, Kobo Editions. p.203-220.

Zun Qiu W, Gui Zhou Y, Qing Ping Z, You Jun J, Kai Yu T, Hua Ping C \& Qian Ming H (2015) Purification, dynamic changes and antioxidant activities of oleuropein in olive (Olea europaea 1.) leaves. Journal of Food Biochemistry, 39:566-574. 